\title{
Rational Modification of a Selection Strategy Leads to Deoxyribozymes that Create Native 3'-5' RNA Linkages
}

Rebecca L. Coppins and Scott K. Silverman*

Department of Chemistry, University of Illinois at Urbana-Champaign, 600 S. Mathews Ave., Urbana, Illinois 61801

Sequences of individual 8AY deoxyribozymes

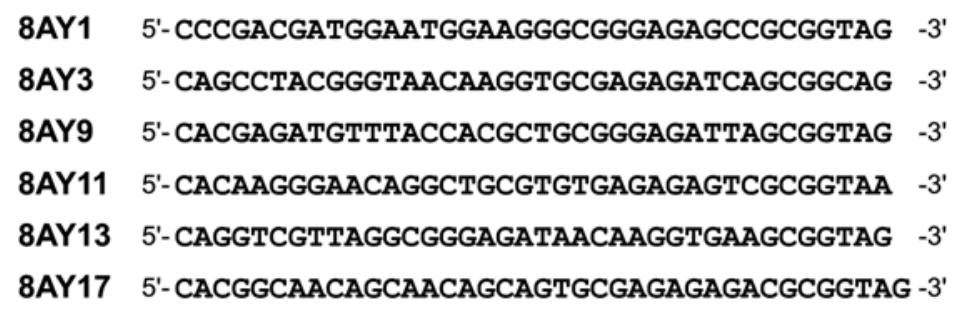

Figure X1. Sequences of the six 8AY deoxyribozymes as determined by automated sequencing. Only the enzyme regions are shown; these were $\mathrm{N}_{38}$ regions at the start of selection. Unlike the other five deoxyribozymes, 8AY17 has a 39-nt enzyme region, likely due to a spurious nucleotide insertion by Taq polymerase during selection. 


\section{$\underline{\text { Representative secondary structures predicted by mfold }}$}

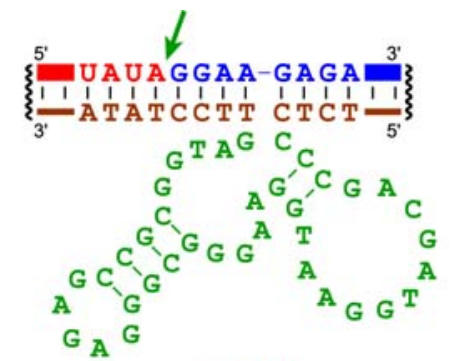

8AY1

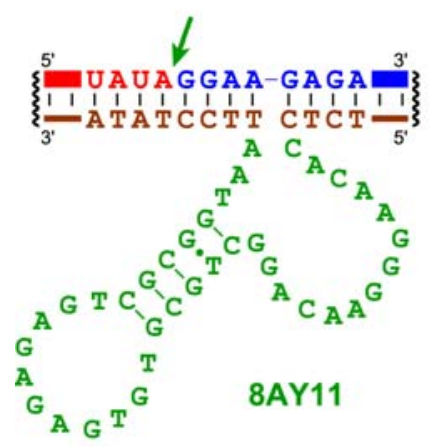

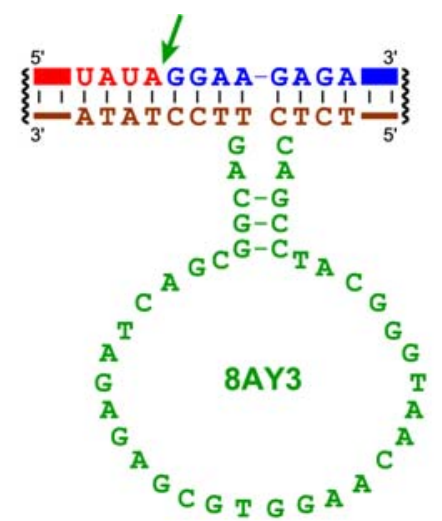
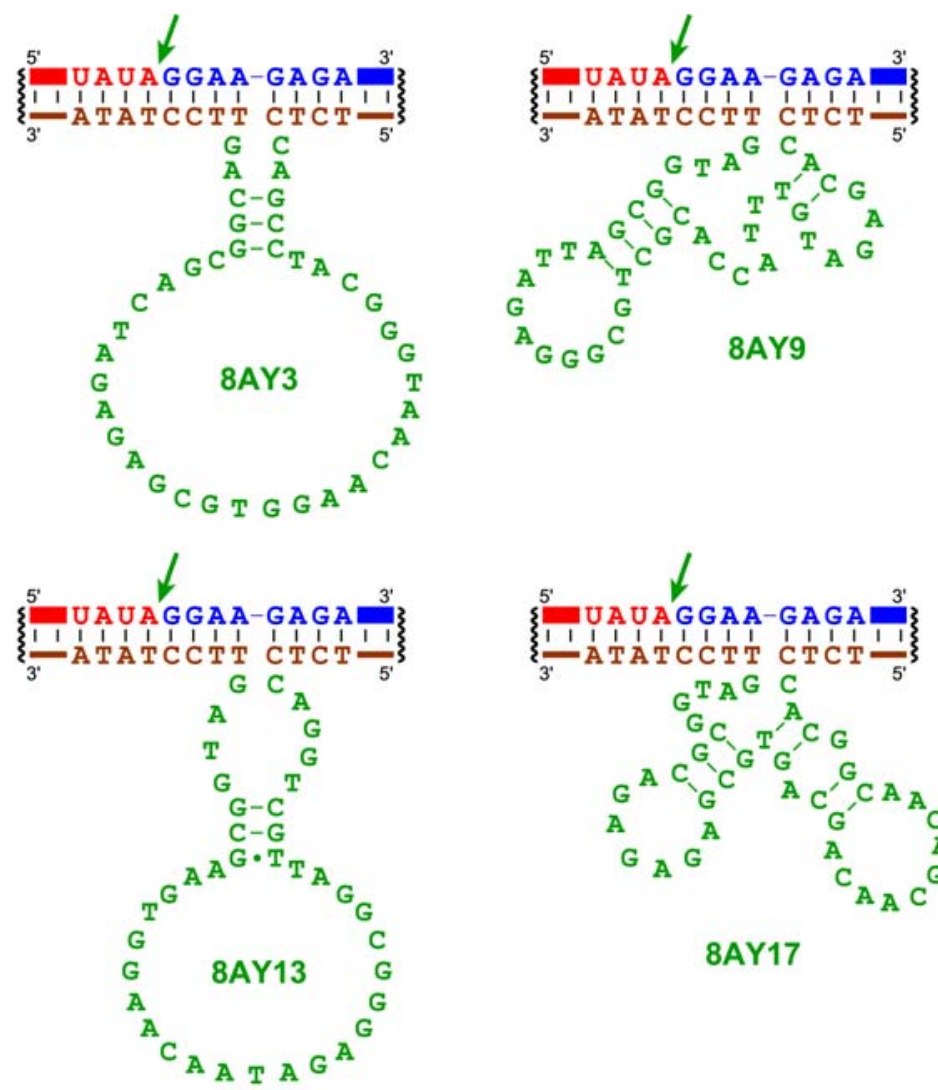

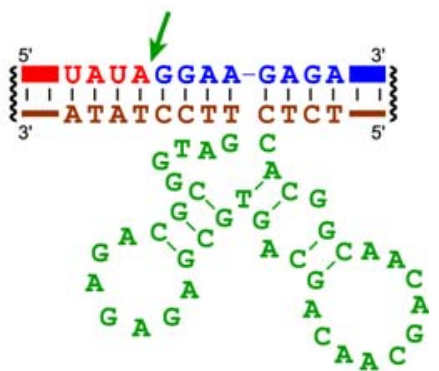

8AY17

Figure X2. Representative secondary structures for the six 8AY deoxyribozymes as predicted by mfold. The upper strands are RNA (red = left-hand substrate, blue = right-hand substrate). The DNA nucleotides are colored brown for the substratebinding arms and green for the enzyme region. All deoxyribozymes retain the RNA/DNA arrangement depicted in strategy 2 of Figure 2C, in which the ligation site (marked by the green arrow) is embedded within an RNA:DNA duplex. The illustrated structures are the lowest-energy mfold-predicted structure for each deoxyribozyme. In some cases, other secondary structures (not shown) are of comparable energy. The DNA version of the mfold algorithm was used. Because mfold is not parameterized for RNA:DNA hybrids and because the structure of the DNA enzyme was the main interest, the substrate strands were input as DNA. Because the results shown above reveal that strict Watson-Crick pairing is maintained for all substrate nucleotides with the corresponding DNA binding arms, this substitution of DNA for RNA is apparently of no consequence for the purpose of showing representative structures like these. A key point is that none of the DNA enzymes has any mutations in the DNA nucleotides near the ligation site, such that the designed arrangement of strategy 2 in Figure 2C is faithfully maintained. 